\title{
Vorschau
}

4 | 2010

\section{Unsere Themen im nächsten Heft}

\section{Bilanzorientiertes Risikoma- nagement in Industrieunter- nehmen}

Dr. Matthias J. Rapp

Die Finanz- und Wirtschaftskrise ist immer noch ein aktuelles Thema. Im Zuge dessen gewinnt das Risikomanagement - wie generell in Krisenphasen - in den Unternehmen zunehmend an Bedeutung. Vor diesem Hintergrund zeigt der Autor in seinem Artikel die rechtlichen Grundlagen des Risikomanagement auf und stellt anhand einer bilanzorientierten Sichtweise dar, wie Risiken in Unternehmen identifiziert, bewertet, gesteuert und kommuniziert werden können.
Die monetäre Bewertung technologiebasierter immaterieller Vermögensgegenstände

Dr. Harald Wirtz

Technologiebasierte immaterielle Vermögensgegenstände stellen wichtige Faktoren für den unternehmerischen Erfolg dar. Für die monetäre Bewertung von Vermögenswerten stehen mit dem Kosten-, dem Markt- und dem Barwertansatz drei grundlegende Herangehensweisen zur Verfügung. Innerhalb jedes Ansatzes lassen sich mehrere Methoden unterscheiden. Diese klassischen Verfahren sind auf Technologien allerdings nur mit gewissen Einschränkungen anwendbar. Harald Wirtz diskutiert in dem Artikel die grundlegenden Bewertungsansätze und -methoden und zeigt ihre Grenzen hinsichtlich der Anwendbarkeit auf technologiebasierte immaterielle Vermögenswerte auf.

\begin{abstract}
Abgrenzung von zahlungsmittelgenerierenden Einheiten im Rahmen des Impairment Test aus dem Blickwinkel der internen Unternehmenssteuerung Prof. Dr. Stefan Müller

Dr. Jens Reinke

Für einen einzelnen Vermögenswert lassen sich dessen Nettoveräußerungswert und Nutzungswert zumeist nicht verlässlich bestimmen. Folge in der Rechnungslegung nach IFRS ist ein Abweichen vom Grundsatz der Einzelbewertung, sodass der erzielbare Betrag auf Ebene einer zahlungsmittelgenerierenden Einheit (ZMGE) zu bestimmen ist. In vielen Fällen bietet sich zur Identifikation von ZMGEs ein Rückgriff auf interne Controllingstrukturen des Unternehmens an, was als IAS 36 spezifische Ausprägung des Management Approach verstanden werden kann. Für die interne Unternehmenssteuerung ergibt sich hieraus die Möglichkeit der Einflussnahme auf die Gestaltung des Jahresabschlusses.
\end{abstract}

